\title{
Are there more differences or similarities between the hospital-acquired pneumonia guidelines?
}

\author{
Andre C. Kali1 ${ }^{1}$, Mark M. Metersky ${ }^{2}$ \\ ${ }^{1}$ Department of Internal Medicine, Division of Infectious Diseases, University of Nebraska Medical Center, Omaha, NE, USA; ${ }^{2}$ Division of \\ Pulmonary, Critical Care, and Sleep Medicine, University of Connecticut School of Medicine, Farmington, CT, USA \\ Correspondence to: Andre C. Kalil, MD, MPH. Professor of Medicine, University of Nebraska Medical Center, 985400 Nebraska Medical Center, \\ Omaha, NE 68198, USA. Email: akalil@unmc.edu.
}

Submitted Oct 07, 2018. Accepted for publication Oct 09, 2018.

doi: $10.21037 / \mathrm{atm} .2018 .10 .28$

View this article at: http://dx.doi.org/10.21037/atm.2018.10.28

The 2016 hospital-acquired (HAP) and ventilatorassociated pneumonia (VAP) guideline was sponsored by the Infectious Disease Society of America (IDSA) and the American Thoracic Society (ATS), and endorsed by the Society of Critical Care Medicine (SCCM), American College of Chest Physicians (CHEST), and the Society for Healthcare Epidemiology (1). The 2017 guidelines for the management of HAP and VAP was sponsored by the European Respiratory Society (ERS), and endorsed by the European societies of Intensive Care Medicine and Clinical Microbiology and Infectious Diseases, and the Latin American Thoracic Association (2). For practical reasons we will use the abbreviations IDSA/ATS and ERS Guidelines throughout the text in which we will compare the sections between both guidelines. While this article will provide the highlights of these guidelines, we hope this will encourage the readers to go through the original documents for a comprehensive reading.

\section{Comparison of guideline construct and methodology}

Both guidelines used the Grading of Recommendations, Assessment, Development, and Evaluation (GRADE) methodology, but the IDSA/ATS only used GRADE recommendations whereas the ERS guideline frequently used "good practice" statements (suggestions based on opinion) instead of making GRADE recommendations (formal assessment based on evidence) for PICO (Patient/ Population, Intervention/Indicator, Comparator/Control, Outcome) questions. The IDSA/ATS guideline had a strict requirement from both sponsoring societies for each guideline panelist to disclose any actual, potential, or perceived conflicts of interest (COI), then the ethical committees of each society decided if the panelist would be accepted or not to participate; further, for the remaining duration of the guideline work until publication release, all panelists had to provide an update of his/her COI for every meeting, which were done in a monthly basis. The ERS guideline did not have the same COI approach in place, disclosing the potential COIs as part of the supplemental publication. The IDSA/ATS guideline included evidence published until December 2015 and performed multiple original statistical frequentist and Bayesian analyses, as well as meta-analyses as an integral part of the panel evaluations and deliberations; the ERS guideline included literature until September 2016 and did not perform any original statistical analysis. The definitions of HAP and VAP were the same for both guidelines; however, in contrast to the 2017 ERS, the 2016 IDSA/ATS guideline formally separated HAP from VAP for both diagnostic and treatment PICO questions. This recognition of two respiratory clinical entities addresses the fact that both the host and the epidemiologic risk factors for MDR (multi-drug resistant) pneumonia are dissimilar between HAP and VAP. Healthcare-associated pneumonia (HCAP) was removed from both guidelines, because HCAP failed to distinguish patients with high risk for MDR pneumonia.

\section{Comparison of diagnostic recommendations}

The IDSA/ATS panelists performed a systematic review 
and meta-analysis to evaluate the clinical outcomes related to invasive $v s$. non-invasive methods, and quantitative $v s$. semiquantitative culture methods; the findings from these several analysis showed no differences in relevant patient outcomes such as mortality, days on ventilation, intensive care unit (ICU) days, or antibiotic changes. Based on this extensive evaluation that found no differences in patient outcomes, plus the higher risk for antibiotic initiation delay and potential safety issues associated with invasive procedures (bronchoscopy), the IDSA/ATS guideline recommends non-invasive modalities with semiquantitative cultures (1). The ERS guideline recommends that either modality be used (2). Neither guideline recommends the use of biomarkers for the diagnosis of HAP/VAP, based on current evidence. The IDSA/ATS guideline recommends both sputum and blood cultures, while the ERS guideline recommends only sputum. The rationale for taking blood cultures is three-fold: (I) the presence of bacteremia provides further evidence of microbial etiology; (II) it increases the probability of using the most appropriate antibiotics; (III) it may lead the physician to non-respiratory sources of infections and better source control if the pathogen is affecting other organs.

\section{Comparison of treatment recommendations}

The IDSA/ATS guideline did not use the concept of early$v s$. late-onset to direct antibiotic therapy. The definition of early onset VAP varies substantially among studies; earlyonset has been defined as first 2 , or 3 , or 4 , or 5 days from hospitalization; and late-onset has been defined as greater than 3 , or 4 , or 5 days). More importantly, evidence has shown that MDR infection, while somewhat less common in early VAP, still occurs in substantial numbers (1); thus, the IDSA/ATS panelists concluded that there was not robust enough evidence to safely support the of use narrow-spectrum antibiotics in early-onset pneumonia. On the other hand, the ERS guideline made specific recommendations for early-onset VAP. This may reflect a more patient-centered approach of the IDSA/ATS guideline, compared to a more public health approach of the ERS guideline concerning treatment recommendations.

Regarding the initial empiric antibiotic treatment, both IDSA/ATS and ERS guidelines emphasize that the knowledge of local antibiograms is relevant for the best choice of empiric antibiotics. Concerning the choice of initial antibiotics, the ERS guideline provides an algorithm based on risk for mortality (>15\%) and MDR infections ( $>25 \%)$, which suggests the use of narrowor broad-spectrum therapies based on different risks; however, this algorithm was not an integral part of a formal recommendation. The IDSA/ATS used a different approach: after performing a systematic review of the literature, the panelists performed multiple statistical analyses to quantify the specific risks for MDR pathogens, and their results demonstrated five risk factors that are more frequently associated with MDR VAP: two epidemiologic factors-prior intravenous antibiotic use within 90 days and 5 or more days of hospitalization prior to VAP; one local factor-hospital and unit microorganism prevalence and susceptibility; and three disease severity factors: septic shock at time of VAP, ARDS preceding VAP, and need for acute renal replacement. Of note, both IDSA/ATS and ERS guidelines recommend initial empiric coverage for Pseudomonas spp. and MRSA (methicillinresistant Staphylococcus aureus) in patients with risk factors for MDR infections and/or admitted to an ICU with a high prevalence of MDR pathogens.

The ERS guideline does not provide any specific recommendation on inhaled antibiotics or on the use of pharmacokinetic/pharmacodynamic (PK/PD) approach to optimize antibiotic dosing, while the IDSA/ATS guideline performed separate systematic reviews and metaanalyses of all studies that evaluated these two therapeutic approaches. The IDSA/ATS panelists' findings led to the recommendation that inhaled antibiotics should be used only in the setting of Gram-negative microorganisms that are sensitive only to aminoglycosides or polymyxins. Similarly, the IDSA/ATS panelists' analyses concluded that the optimization with $\mathrm{PK} / \mathrm{PD}$ was associated with improvements in mortality, clinical cure, and ICU length of stay (1). Subsequently, several studies further corroborated the IDSA/ATS PK/PD recommendation (3-5).

\section{Comparison of length of antibiotic therapy}

Both IDSA/ATS and ERS guidelines recommend 7-8 days of antibiotic therapy for most patients, however, the ERS panelists noted that short-course therapy "may not be possible" for non-fermenting Gram-negative pneumonias. In contrast to the ERS guideline, the IDSA-ATS guideline panel performed a systematic review and meta-analyses on all nosocomial pneumonias, as well as on specific subsets of patients with and without non-fermenting Gram-negative pneumonias, and their findings consistently showed no differences for the following outcomes: mortality, clinical 
cure, recurrent pneumonia, and mechanical ventilation duration. Further, the IDSA-ATS panelists recognized that shorter or longer courses may be needed for a minority of patients depending upon "the rate of improvement of clinical, radiologic, and laboratory parameters", and this was valid for any patient independent of the microbial etiology. The ERS guideline also recommended longer antibiotic courses for patients with lung abscess or necrotizing pneumonia, but these two clinical syndromes are expected to have prolonged course of symptoms and slow clinical recovery, so they also fall within the IDSA/ATS specific remarks to provide longer therapy based on individual patient response.

\section{Comparison of antibiotic de-escalation}

The IDSA-ATS guideline recommends that procalcitonin may be used to de-escalate antibiotic therapy in patients with VAP based on the panelists' meta-analysis of three randomized controlled trials. More recently, two new studies gave further support for this IDSA/ATS recommendation $(6,7)$. The ERS guideline suggests the use of procalcitonin in settings that may require longer antibiotic courses, such as the severe immunocompromised, pneumonia due to highly antibiotic resistant microorganisms, and need for second-line antibiotic therapy; however, the use of procalcitonin has not been studied in any of these settings.

\section{Conclusions}

Two guidelines have been published in the last two years for the diagnosis and treatment of patients with HAP and VAP. It is remarkable that despite using similar literature sources, both guidelines resulted in several different recommendations. Possible explanations for that include the following: the uncertainty related to the low to moderate quality of evidence, which resulted in more subjectivity that is inherently more dependent on experts' opinions; the clinical practice variations associated with different geographic areas; the distinct set of hospital-residing bacteria among various countries and local institutions; and the more structured methodology with the performance of numerous formal statistical analyses by the IDSA/ATS panel. Nonetheless, when both guidelines are reviewed as a whole, there are clearly more similarities than differences between them. Both guidelines' group of panelists had the same goal, i.e., to improve the outcome of all patients with HAP and VAP. We hope their efforts will pave the way to advance clinical care and spur more research in the field of HAP and VAP.

\section{Acknowledgements}

None.

\section{Footnote}

Conflicts of Interest: The authors have no conflicts of interest to declare.

\section{References}

1. Kalil AC, Metersky ML, Klompas M, et al. Management of Adults With Hospital-acquired and Ventilator-associated Pneumonia: 2016 Clinical Practice Guidelines by the Infectious Diseases Society of America and the American Thoracic Society. Clin Infect Dis 2016;63:e61-e111.

2. Torres A, Niederman MS, Chastre J, et al. International ERS/ESICM/ESCMID/ALAT guidelines for the management of hospital-acquired pneumonia and ventilator-associated pneumonia: Guidelines for the management of hospital-acquired pneumonia (HAP)/ ventilator-associated pneumonia (VAP) of the European Respiratory Society (ERS), European Society of Intensive Care Medicine (ESICM), European Society of Clinical Microbiology and Infectious Diseases (ESCMID) and Asociacion Latinoamericana del Torax (ALAT). Eur Respir J 2017;50:1700582.

3. Abdul-Aziz MH, Sulaiman H, Mat-Nor MB, et al. BetaLactam Infusion in Severe Sepsis (BLISS): a prospective, two-centre, open-labelled randomised controlled trial of continuous versus intermittent beta-lactam infusion in critically ill patients with severe sepsis. Intensive Care Med 2016;42:1535-45.

4. Roberts JA, Abdul-Aziz MH, Davis JS, et al. Continuous versus Intermittent beta-Lactam Infusion in Severe Sepsis. A Meta-analysis of Individual Patient Data from Randomized Trials. Am J Respir Crit Care Med 2016;194:681-91.

5. Vardakas KZ, Voulgaris GL, Maliaros A, et al. Prolonged versus short-term intravenous infusion of antipseudomonal beta-lactams for patients with sepsis: a systematic review and meta-analysis of randomised trials. Lancet Infect Dis 2018;18:108-20.

6. de Jong E, van Oers JA, Beishuizen A, et al. Efficacy and safety of procalcitonin guidance in reducing the 
duration of antibiotic treatment in critically ill patients: a randomised, controlled, open-label trial. Lancet Infect Dis 2016;16:819-27.

7. Schuetz P, Wirz Y, Sager R, et al. Effect of procalcitonin- guided antibiotic treatment on mortality in acute respiratory infections: a patient level meta-analysis. Lancet Infect Dis 2018;18:95-107.

Cite this article as: Kalil AC, Metersky MM. Are there more differences or similarities between the hospital-acquired pneumonia guidelines? Ann Transl Med 2018;6(21):429. doi: 10.21037/atm.2018.10.28 